\title{
The Mental Representation of Occupational Stereotypes is Driven As Much By Their Affective As By Their Semantic Content
}

Ferenc Kocsor ( $\sim$ kocsor.ferenc@pte.hu )

University of Pecs

Tas Ferencz

University of Pecs

Zsolt Kisander

University of Pecs

Gitta Tizedes

University of Pecs

Blanka Schaadt

University of Pecs

Rita Kertész

University of Pecs

Luca Kozma

University of Pecs

Orsolya Vincze

University of Pecs

András Láng

University of Pecs

\section{Research Article}

Keywords: stereotypes, representations, person perception, categorization

Posted Date: October 25th, 2021

DOl: https://doi.org/10.21203/rs.3.rs-956124/v1

License: (1) (i) This work is licensed under a Creative Commons Attribution 4.0 International License.

Read Full License 


\section{Abstract}

Background: Studies on person perception showed that stereotypes can be activated by presenting either characteristic traits of group members, or labels associated to these groups. However, it is not clear whether these pieces of semantic information activate negative and positive stereotypes directly, or via an indirect cognitive pathway leading through brain regions responsible for affective responses. To disentangle the effects of semantic and affective contents, first we intended to scrutinize whether the representation of occupational labels is independent of the emotions they evoke.

Methods: Participants ( $N=71, M=27.0, S D=9.1,31$ men 42 women,) were asked to complete two tasks presented online. In the first task they had to arrange 20 occupational labels-randomly chosen from a pool of 60 items - in a two-dimensional space, moving the mouse pointer along two undefined axes. In a second task the axes' names were defined a priori. Subjects were asked to arrange the labels according to valence, the extent to which the word evoked pleasant or unpleasant feelings, and arousal, the extent to which the word evoked excitement or calmness.

Results: Based on the final coordinates of the labels, two cluster analyses were carried out separately in the two tasks. The two clusters were compared with Fisher's exact test, which revealed that the cluster structures overlap significantly.

Conclusions: The results suggest that the semantic representation of occupations relies largely on the affective state they evoke. We propose that affective content might have a primacy over detailed semantic information in many aspects of person perception, including categorization and face recognition.

\section{Background}

\subsection{Stereotypes evoked by occupational labels}

The term stereotype refers to a generalized and simplified belief about a group of people (1), which forms through a socially embedded cognitive process that involves associating attributions to the group (2). It is widely accepted that stereotypes might be formed based on direct observations [i.e. data-driven model of stereotypes, e.g., (3)], or expectations held of a group [theory-driven model, e.g., (4)], or reflect a combination of the two models (5). It extends to physical appearance, interest, occupations or any similar characteristics held by a group of people (6-9). Since categorization is an inherent part of all perception (10), stereotypes are activated automatically along the perception of an individual (11-17). In this process a few attributes typical for a group are sufficient to infer group membership and behavioral characteristics. In addition to gender, age, ethnicity, beauty, and other stereotypes, occupational labels also activate automatic inference processes, and elicit the overall representation of a particular group (18). 
While the content of stereotypes may differ between cultures, several principles of stereotyping are culturally universal (19). In a study conducted in the USA and Germany, participants were asked to form groups of listed professions (20). A striking convergence was found between the stereotype dimensions in the categorization in the two countries. Participants of the study predominantly discriminated occupations based on agency and progressiveness, and to some extent on sociability as well. Further research discovered that the employees were not only identified by their job title, but assumed personality traits were also assigned to them based on their professions. It was also noticed that positive or negative perceptions of occupations in the same group were "transferred" to the rest of the group, so that the groups also received a kind of shared rating (20). The conclusion is the same as in most previous studies on stereotypes (21-23): if we do not have enough information about a person as an individual, inference will be based on categorical attributes.

\subsection{Semantic processing in person perception}

One of the most systematic studies to date which aimed to map how semantic information, delivered by occupational labels, is represented, was made by (20). As noted in the previous section, their research focused on those possible dimensions that could serve as anchors when evaluating people. Undeniably, the number of dimensions and variations of spontaneous categorization could be countless. Therefore, the study focused on filtering out the most typical and practical ones. To obtain truly spontaneous stereotypes that best reflect what people usually think of first, the participants were free to come up with dimensions based on their own logic, and to classify and categorize the target labels according to these dimensions. The researchers found that the most frequent dimension by choice was agency. For instance, surgeons, software developers, and aerospace engineers were placed the top of this dimension; cashiers, telemarketers, and parking attendants at the bottom. This suggests that agency refers to being powerful, assertive, and high in status. The second most frequent defining dimension was progressiveness. On this dimension, paramedics, firefighters, and police officers were labelled as conservative, conventional, and preventive (rule-based) types, while musicians, athletes, and designers were more likely to be labelled as liberal, alternative, and promotional types (characterized by innovation, risk-taking, brainstorming, etc.) $(20,24)$.

One obvious result of the study above is that it has shown that different occupations evoke similar stereotypes quite consistently. Certain social expectations may come with these resulting dimensions, which in turn can also be associated with evaluative judgements. However, beyond the fact that some abstract categorization happens, we do not know much about the basic cognitive processes underlying these attributions.

Much of what we know about the cognitive processes of person perception comes from studies on the cognitive background of face perception. Bruce and Young's (25) cognitive model, later improved by Breen et al. $(26,27)$, shows that a key element of intact, conscious recognition of persons is that faces should also recall semantic information. These include names, occupations, places of residence, and so 
on. The so-called person identification node integrates visual information from the face with knowledge stored in memory. At the same time, characteristics that are not very specific to the individual-but are nevertheless important attributes, such as the aforementioned dimensions of agency, progressiveness, and sociability-might be parts of the features that are also involved in person recognition, just like they are elements of semantic information processing. Although the latter has not been investigated directly, there have been several concordant studies that show that facial appearance is used for spontaneous inference about trustworthiness and competence within a short time (28-31).

\subsection{Affective processing during face encoding - a model for understanding abstract representations}

Just as stereotypes are based on observed behavior and typical physical appearance, stereotypical descriptions activate representations of both expected behavior and physical appearance. Therefore, we need to examine at which level evaluation of individuals happens in the first place: is it appearance, behavior, or semantic knowledge? Each of these evoke affections directly, and the provided emotional content in turn contributes to the conscious recognition of persons, as it has been implied by the most influential face perception models $(26,32,33)$.

The concept of affective space is anchored to face recognition models $(7,8)$, and is also present in research on person perception $(34,35)$. Affective space is considered as a two-dimensional categorization system, where faces are assigned a place based on the emotions they evoke in the perceiver (36). The dimensions of affective space are arousal (intensity) and valence (pleasantness). These dimensions can be thought of as the $X$ and $Y$ axes of a coordinate system where each face has a valence-arousal coordinate. In the dimensional approach, the intensity of the emotions we experience ranges, along the vertical axis, from low activation to high alertness (i.e., from calm to agitated, or bored to tense), while on the horizontal axis of the dimension the valence ranges from negative to positive (i.e., unpleasantpleasant, sad-satisfied, upset-joyful). Valence refers to a kind of evaluation, or value attribution, that is subjectively induced by the appearance of emotions, while arousal refers to the level of activation or energization associated with emotions and their physiological characteristics $(37,38)$. The study of Lang (37) explains emotional valence and arousal in terms of the functioning of specific motivational systems of the brain. According to the dimensional approach, the neural functioning of the two is mainly determined by valence, i.e., the emotional evaluation itself (38). Valence is associated with the functioning of the two types of motivational systems in the brain and therefore plays a primary role (37). According to Bradley and colleagues (36), the affective space can be equated with the approachavoidance system, i.e. the appetitive and the aversive systems. The appetitive system is associated with pleasant things (exploratory behavior, eating, sexual behavior), but their intensity can vary from a relaxed state to an aroused state; while the aversive system deals with unpleasant consequences (avoidance, defensive behavior), also showing a large variance along the arousal. The latter, since it communicates only differences in activation to the appetitive, the aversive, or both systems, has only a secondary, complementary role in the dimensional ordering of emotions (37). 
The affective content elicited by unfamiliar faces depends primarily on the structural features of the face, its attractiveness, and how it is categorized by the observer. In person perception research, prejudices are typical cases of the expression of emotions. For someone who is prejudiced against a race, the perception of characteristic physical traits of that race is accompanied by the affective content of the prejudice (39). The emotions evoked by the face of personal acquaintances and famous people, in contrast, depend on the specific experiences associated with that individual (40-43). According to cognitive models of face perception, affective content plays an essential role in the conscious recognition of persons, in addition to semantic content. If the affective processing pathway is impaired, it leads to severe face recognition deficits. For instance, patients with Capgras-syndrome are able to recognize people who they met before, however, the impairment of the so called covert pathway of face recognition - which is responsible for affective contents - prohibits the acceptance of the fact that the observed faces are identical to those who are personally familiar $(26,32)$. In contrast, patients suffering from prosopagnosia (i.e., an inability to consciously recognize familiar faces) show elevated physiological arousal (including increased heart rate and galvanic skin response) when they are exposed to faces of close acquaintances. In these individuals the overt pathway of face recognition is impaired, whereas the covert pathway is intact $(26,27,44)$. In summary, neuroanatomical models of face recognition suggest that a sufficient affective charge is necessary for the activation of the faces stored in the memory. Similarly, when approaching the process of face recognition from a representational view, we can say that stimulation of the affective space activates the associated region of the face space. Such affective content can be conveyed by labels that are known to activate stereotypes, that is which evoke our expectations of behavior and appearance.

\subsection{Interconnectedness of representational spaces}

The process of face recognition combines semantic and affective information to allow the recall of familiar faces. Above, we briefly summarized how this happens on the neural level. However, relatively little research has explored the interconnectedness of representational spaces, or tried to integrate the available pieces of information into a cognitive model. One notable attempt, called the Trait Inference Mapping (TIM) model (45), aimed to combine the concepts of face space with trait space.

However, this model has a rather monolithic view of trait space, and does not differentiate between the semantic and the affective contents that constitute the representation of traits. Furthermore, it only focuses on the physical aspect of people, namely faces. Nevertheless, the presentation of a face leads very rapidly to categorization, which in turn activates stereotypes related to that category. Hence, TIM is a good example of how different representational spaces might interact. Taking advantage of the approach of TIM, we aim to extend and generalize this model by suggesting that any aspect of a person-including facial appearance, group belonging, typical behavior, occupation, or any other characteristics-can be treated as an individual representational space, and thus be the subject of analysis. Similarly, trait space might be divided into semantic and affective spaces, and the latter one, if necessary, broken down to indices of valence and arousal. It is important to note that this approach serves the aims of a theoretical 
investigation; it is an abstract, purely cognitive model, and might not persevere when its neuroanatomical implications are tested.

\subsection{Aims and hypotheses}

Person perception can be understood as a process of integrating different representational spaces. In this process, the image of each person is composed of elements that sometimes complement each other and sometimes mutually determine each other. Examples of such elements are position in face space, i.e. physical appearance (including facial symmetry, masculinity/femininity, skin texture, etc.), semantically interpretable attributes (group category, occupation, etc.), and position in affective space, determined by valence and arousal. These components may be interpreted at different levels of neural processing, but they can nevertheless be incorporated into a common cognitive model. In our first attempt to build a usable model of person perception, we focus on the processing of semantic and affective information. Therefore, the fundamental question of our research was to explore how big of a role the semantic content of labels, as well as the emotional responses they elicit, play in the processing of group-typical labels.

To this end, we designed an experiment where participants had to arrange occupational labels based on their semantic and their affective contents, respectively. Our analysis plan was to run cluster analyses on the arrangements to see whether the labels grouped together in the first task would show the same pattern in the second task as well. This analysis would reveal some of the connections between the two representational spaces. We expected that the representational space of affective contents shows a significant overlap with the space of semantic representations.

\section{Methods}

\subsection{Participants}

Our online study consisted of two tasks. Seventy-three people participated in the first task $(M=27.0$; $S D=9.1$, $\min =18 ; \max =58): 31$ men $(M=27.9 ; S D=10.3$, $\min =18 ; \max =56)$ and 42 women $(M=26,4 ; S D=8,3$; $\min =18 ; \max =58)$. Their ages ranged between 18 and $58(M=26.9 ; S D=8.96)$. From this sample, 52 people completed the second part as well $(M=25.6 ; S D=8.5, \min =18 ; \max =58): 24$ men $(M=26.8 ; S D=9.4$, $\min =20 ; \max =56)$ and 28 women $(M=24.6 ; S D=7.7 ; \min =18 ; \max =58)$. Demographic data were analyzed using Jamovi 1.6.23 $(46,47)$.

\subsection{Procedure}

We selected 53 occupational labels from the pool used by (20), and translated these into the native language of the participants. Seven additional labels, which referred to criminal activity instead of occupations (e.g. drug dealer, mob member, thief, etc.), were added to the item pool in order to expand it 
with low valence labels (Appendix, Table App1). The two experiments were programmed in Javascript, and data were collected using Qualtrics.

In the first task, participants were asked to arrange 20 labels on the screen, using the Spatial Arrangement Method paradigm $(24,48)$. The labels were randomly selected from the 60 -item pool for each of the participants, and appeared on the screen, in a random arrangement, in four columns and five rows. After presenting the 20 labels together, participants were exposed to these consecutively one at a time during the task. They were instructed to arrange the labels on the screen with the mouse pointer, and use any aspects which they thought would adequately differentiate between labels. That is, occupational labels perceived as similar were more likely to be placed close to each other than labels evoking dissimilar concepts about the people they refer to. After completing the task, participants were also asked to report what name they would have given to the axes.

The second task was very similar to the first one, the only difference being that the participants had to arrange the labels along pre-specified dimensions. Specifically, it was explained that the $\mathrm{X}$-axis refers to the extent to which each word evokes pleasant or unpleasant feelings (i.e, valence), and the $Y$-axis represents the extent to which the word evokes excitement or calmness (i.e., arousal). The endpoints of the axes were marked with adjectives that described the evoked states, such as sadness, anger, fear, depression (far left), happiness, good feeling, contentedness, hope (far right), excitement, awareness, enhanced attention (top), and calmness, boredom, and sleepiness (bottom), respectively. Hence, for example, labels that evoked positive feelings and excitement were expected to be clustered together in the top right-hand quarter of the screen.

\subsection{Data processing}

Based on the coordinates of the labels in the individual responses, the relative semantic distance between the occupations were calculated, using $R$ 4.1.0 (49), with the following formula:

relative distance (label_1; label_2 $)=\left(\right.$ sqrt $\left.\left(\left((\text { label_1_x }- \text { label_2_x })^{\wedge} 2\right)+(\text { label_1_y }- \text { label_2_y })^{\wedge} 2\right)\right)$ / maxdist,

where label_1 and label_2 are the occupations the distance of which are being calculated, label_1_x, label_1_y, label_2_x, and label_2_y are the $X$ and $Y$ coordinates of the labels, and maxdist is the maximal possible distance between two labels on the screen (i.e., the diagonal of the task area). After the standardized Euclidean distance of each pair had been calculated, each was averaged across all participants who had repositioned the two occupations. Then we transformed the relative distances into triangular form to allow multidimensional scaling with an ALSCAL procedure (50) using the Matrix package in R (51). It has been shown (20) that scaling stress fit is satisfying both in terms of scaling stress and parsimony for a 3 dimensional model. Therefore, we calculated the coordinates of each label for a 3D space using multidimensional scaling. Thus, we received three coordinates for each label. 
Coordinates collected in the second task were processed the same way: calculation of relative distances, averaging across participants, and multidimensional scaling.

\section{Results}

\subsection{Comparison of cluster content}

We ran cluster analyses on the coordinates of both the semantic and the affective spaces, respectively, with the Ward-method in SPSS 26. The visual inspection of the dendrograms suggested that the labels were positioned by the participants into five distinct clusters in both spaces (Appendix, Fig. App1 and Fig. App2). For each semantic cluster we calculated the number of those labels which were positioned into that cluster, while at the same time into one particular affective cluster. We did the same for each semantic-affective cluster combination. For instance, sailors and police officers were grouped in the first cluster in the affective space (A1) and the first cluster in the semantic space (S1), therefore the value of the respective cell (i.e., A1/S1) was 2 . There was only one label (bookkeeper) in the fifth affective cluster (A5), which, at the same time, belonged to the fourth semantic cluster (S4). Hence, its cell count was 1 (Table 1).

Table 1. Number of labels belonging to each cluster pair

\begin{tabular}{llllllll} 
Cluster No. & \multicolumn{4}{c}{ semantic clusters } & \multicolumn{2}{c}{ Total } \\
\cline { 2 - 7 } & & S1 & S2 & S3 & S4 & S5 & \\
\hline \multirow{2}{*}{ affective clusters } & A1 & 2 & 0 & 9 & 7 & 0 & 18 \\
\cline { 2 - 6 } & A2 & 0 & 2 & 0 & 0 & 5 & 7 \\
\cline { 2 - 6 } & A3 & 4 & 0 & 3 & 8 & 0 & 15 \\
\cline { 2 - 6 } & A4 & 0 & 0 & 2 & 0 & 0 & 2 \\
\hline Total & A5 & 9 & 0 & 8 & 1 & 0 & 18 \\
\hline & & 15 & 2 & 22 & 16 & 5 & 60
\end{tabular}

To reveal whether the distribution of the content of the affective clusters are independent from the distribution in the semantic space, we ran Fisher's exact test using Monte Carlo simulation in $R$ (49). For instance, if all values in Table 1 would be zeros, except one cell in each row and each column (for example the cells in the diagonal), it would mean that location of the occupational labels in the affective space determines their position in the semantic space as well. In contrast, if the numbers in the cells would be distributed evenly or arbitrarily, there would be no non-random overlap in the positioning of the labels in the two spaces. However, the simulated p-value of Fisher's exact test, based on $1 \mathrm{e}+07$ replicates, showed that the arrangement of the labels in the two tasks was significantly different from a fully 
independent one $(p=.0000001)$. The value of Cramer's $V$ was 0.57 , which also confirmed that the two cluster analyses were similar to each other to a large extent.

\subsection{Categorization of axis labels}

To receive an overview of the kind of representations the individual labels might have evoked in the participants when they had a free choice to arrange them, the labels provided by the participants in the first task were grouped, categorized, and their frequency of occurrence analyzed (Table 2). Though no clear correspondence could be detected, the received categories suggest that the $2 \mathrm{D}$ dimensions obtained with semantic arrangement align with those dimensions developed to characterize occupational roles by (20). For instance, agency is one of the main factors, which is reflected in our study by the frequent use of axis names that refer to knowledge, physical/intellectual work, and income. Labels categorized along the interesting/attractive occupational axis might carry similar meaning as the dimension of progressiveness. Another dimension, sociability, appears in our responses as axis types such as useful for the society and social desirability.

Table 2. Frequencies of given axis names within label categories

\begin{tabular}{llll} 
Label categories & Counts & $\%$ of Total & Cumulative \% \\
\hline knowledge & 17 & $18 \%$ & $18 \%$ \\
\hline useful for the society & 15 & $16 \%$ & $35 \%$ \\
\hline physical/intellectual work & 12 & $13 \%$ & $48 \%$ \\
\hline income & 15 & $16 \%$ & $64 \%$ \\
\hline social desirability & 15 & $16 \%$ & $80 \%$ \\
\hline interesting/attractive & 12 & $13 \%$ & $93 \%$ \\
\hline other & 6 & $7 \%$ & $100 \%$
\end{tabular}

\section{Discussion}

In the present study, we aimed to investigate the representational level at which stereotypes are formed about people. More specifically, whether the semantic content of stereotypes, that can be activated by labels (i.e., the references to the person's appearance and behavioral characteristics), is an additional required factor in the categorization process, or whether the affective information evoked is sufficient, on its own, for categorization. To do so, participants had to arrange labels describing occupations in a twodimensional space, first in a completely free semantic space, and then according to the elicited valence and arousal, that is, in the affective space. Since stereotype-activating labels will elicit some kind of 
affective response in any case, we expected that there would be an overlap between the two types of grouping. However, it is difficult to quantify the extent of the overlap. The only conclusion that can be drawn from the results of the analyses is that the groupings are not independent of each other. This cannot be attributed to the fact that the same participants were involved in both tasks, as the 20 labels to be clustered in each task were mostly different due to random selection from a 60-item pool.

Nevertheless, the results show that the participants in the experiment grouped the labels similarly in both situations. This suggests that the elicited emotional response already marks the place of the labeled occupations in the representational space. The additional information carried by the labels that more closely describes the behavioral patterns we can expect from the people in the categories that the labels designate, does not in essence modify the representational map.

The results obtained are consistent with the predictions of theories that attempt to account for the formation of stereotypes associated with individuals at the level of representational spaces, but also go beyond to further clarify the cognitive processes that govern this. Over and Cook's (2018) TIM model, for example, conceptualizes the process of categorization as a mapping between regions of face space and regions of trait space. Similarly, Kocsor and Bereczkei (7) found that, under experimental conditions, short behavioral descriptions assigned to individual faces can be used to shape the information stored about trustworthy and untrustworthy faces. Similar results were obtained with purely affectively loaded images that were socially irrelevant and uninformative in terms of semantic content (8). This implies that both semantic and affective content can be generalized and they underlie an essentially affect-based decision. These findings are in compliance with the view we suggested in the introduction (2.4) that processing of information about people happens by mapping elements of numerous representational spaces onto each other: representation of physical appearance, behavioral characteristics, semantic knowledge, and affective information, all of which can be further broken down into overlapping layers of representations.

An analysis of the axes names given by the participants in the first task (Table 2) shows that the considerations in semantic clustering are mostly similar to those obtained in previous research (20). Agency, progressiveness and sociability seem to be dominant aspects when it comes to typifying people and their associated occupational labels. The clusters created under these axes names were not different from those created under valence and arousal. Social utility, social desirability, and appeal have an obvious affective content. This is not the case for expertise, the time and effort required to acquire knowledge, or the physical or mental nature of the work. Nevertheless, the overlap of the clusters that emerge from the two different approaches shows that these contents, eventually, involve similar affective distinctions as the representations based on the valence and arousal induced by the labels. The semantic dimensions used for sorting are largely determined by the underlying affective content of the labels.

As noted above, the differences between the two clusters are difficult to quantify, so in this exploratory study we have merely sought to show that, although the categorization of individuals may be based on information of varying detail, the level of affective representation has already induced a sufficient level of stereotypical categorization. As a future prospect, it would be worth testing empirically whether the overlap between semantic and affective clustering actually influences behavior. In their study, Hills et al. 
(18) found that when faces are associated with labels that do not match their meaning, the time required for recognition is lengthened. Our current study implies that if discrimination based on meaning is essentially identical to discrimination based on elicited emotion, then we would expect equivalent results in a similar reaction time measurement task. That is, we can expect that incongruent priming stimuli (i.e., false labels) will produce roughly similar differences in face recognition speed, regardless of whether they differ semantically or affectively from what is stereotypically expected. If this is not the case, and semantic differences indeed cause a stronger increase in reaction times, then we need to reevaluate our results above and refine our model of person representation.

The latter is necessary in order to reconcile the theoretical approach of this study with the neuroanatomical models of person perception. The latter are mostly concerned with face recognition (e.g., 26,27), but also include the specification of the brain areas involved in the categorization of people. However, in addition to further empirical work, a theoretical integration that combines social psychological theories describing social decisions, cognitive models of representations, and neuroanatomical knowledge of person recognition and categorization is certainly needed. Although our present study is far from being a decisive step in this process, we hope that it will help to pinpoint the cornerstones of a more accurate theoretical approach of person perception.

\section{Declarations}

\section{Ethics approval and consent to participate:}

All methods were performed in accordance with the Declaration of Helsinki and with the relevant guidelines and regulations of the United Ethical Review Committee for Research in Psychology, Hungary, which reviewed and approved the project proposal (ref. Number 2019-120). The participants provided their written informed consent to participate in this study.

\section{Consent for publication:}

Not applicable.

\section{Availability of data and materials:}

The datasets for this study, including SPSS data table and output file can be found in the Open Science Framework repository (https://osf.io/2d7t9/).

\section{Competing interests:}

The authors declare that the research was conducted in the absence of any commercial or financial relationships that could be construed as a potential conflict of interest.

\section{Funding:}

This work, with the lead of Ferenc Kocsor as the PI, was financially supported by the Ministry for Innovation and Technology, Hungary (grant number "NKFI 131411"). Blanka Schaadt was supported by the ÚNKP-2020 New National Excellence Program of the Ministry for Innovation and Technology, 
Hungary, from the source of the National Research, Development and Innovation Fund (grant number "ÚNKP-20-1-I-PTE-820").

\section{Authors' contributions:}

Ferenc Kocsor conceived the study design. Ferenc Kocsor wrote the draft of the manuscript. Ferenc Kocsor and András Láng discussed the statistical analyses, Ferenc Kocsor carried out the analyses. Gitta Tizedes, Blanka Schaadt and Rita Kertész contributed to data collection. Zsolt Kisander and Tas Ferencz made the code for data arrangement and preprocessing. Orsolya Vincze, Luca Kozma and András Láng helped prepare the final version of the manuscript, Ferenc Kocsor finalized the layout, András Láng and Luca Kozma proof-read the manuscript. All authors read and approved the final manuscript.

\section{Acknowledgments:}

The authors are grateful to Roland Imhoff, Johannes Gutenberg Universität Mainz, for the Javascript code that was used for data collection, and for his useful suggestions on data processing.

\section{References}

1. Allport GW. The nature of prejudice. Cambridge: MA: AddisonWesley; 1954.

2. Semin GR. Stereotypes in the Wild. In: Stereotype Dynamics. Psychology Press; 2007.

3. Hamilton DL, Gifford RK. Illusory correlation in interpersonal perception: A cognitive basis of stereotypic judgments. J Exp Soc Psychol. 19760 1;12(4):392-407.

4. Hamilton DL, Rose TL. Illusory correlation and the maintenance of stereotypic beliefs. J Pers Soc Psychol. 1980;39(5):832-45.

5. Haslam SA, Oakes PJ, Reynolds KJ, Turner JC. Social Identity Salience and the Emergence of Stereotype Consensus. Pers Soc Psychol Bull. 1999 Jul 1;25(7):809-18.

6. Hilton JL, von Hippel W. Stereotypes. Annu Rev Psychol. 1996;47(1):237-71.

7. Kocsor F, Bereczkei T. First Impressions of Strangers Rely on Generalization of Behavioral Traits Associated with previously Seen Facial Features. Curr Psychol. 2017 Sep 1;36(3):385-91.

8. Kocsor F, Bereczkei T. Evaluative conditioning leads to differences in the social evaluation of prototypical faces. Personal Individ Differ. 2017 Jan;104:215-9.

9. McGarty C, Yzerbyt VY, Spears R, editors. Stereotypes as explanations: The formation of meaningful beliefs about social groups. Vol. x. New York, NY, US: Cambridge University Press; 2002. 231 p.

10. Bruner JS. On perceptual readiness. Psychol Rev. 1957;64(2):123-52. 
11. Biernat M, Manis M, Nelson TE. Stereotypes and Standards of Judgment. J Pers Soc Psychol. 1991;60(4):485-99.

12. Bodenhausen GV, Wyer RS. Effects of stereotypes in decision making and information-processing strategies. J Pers Soc Psychol. 1985;48(2):267-82.

13. Duncan BL. Differential social perception and attribution of intergroup violence: Testing the lower limits of stereotyping of Blacks. J Pers Soc Psychol. 1976;34(4):590-8.

14. Kunda Z, Sherman-Williams B. Stereotypes and the construal of individuating information. Pers Soc Psychol Bull. 1993;19(1):90-9.

15. Sagar HA, Schofield JW. Racial and behavioral cues in Black and White children's perceptions of ambiguously aggressive acts. J Pers Soc Psychol. 1980;39(4):590-8.

16. Secord PF, Bevan W, Katz B. The Negro stereotype and perceptual accentuation. J Abnorm Soc Psychol. 1956;53(1):78-83.

17. Vallone RP, Ross L, Lepper MR. The Hostile Media Phenomenon. Biased Perception and Perceptions of Media Bias in Coverage of the Beirut Massacre. J Pers Soc Psychol. 1985;49(3):577-85.

18. Hills PJ, Lewis MB, Honey RC. Stereotype priming in face recognition: Interactions between semantic and visual information in face encoding. Cognition. $2008 \mathrm{Jul} ; 108(1): 185-200$.

19. Cuddy AJC, Fiske ST, Kwan VSY, Glick P, Demoulin S, Leyens J-P, et al. Stereotype content model across cultures: Towards universal similarities and some differences. Br J Soc Psychol. 2009;48(1):1-33.

20. Imhoff R, Koch A, Flade F. (Pre)occupations: A data-driven model of jobs and its consequences for categorization and evaluation. J Exp Soc Psychol. 2018 Jul;77:76-88.

21. Krueger J, Rothbart M. Use of categorical and individuating information in making inferences about personality. J Pers Soc Psychol. 1988;55(2):187-95.

22. Liberman Z, Woodward AL, Kinzler KD. The Origins of Social Categorization. Trends Cogn Sci. 2017 Jul 1;21(7):556-68.

23. Shutts K, Roben CKP, Spelke ES. Children's Use of Social Categories in Thinking About People and Social Relationships. J Cogn Dev. 20130 1;14(1):35-62.

24. Koch A, Imhoff R, Dotsch R, Unkelbach C, Alves H. The ABC of stereotypes about groups: Agency/socioeconomic success, conservative-progressive beliefs, and communion. J Pers Soc Psychol. 2016;110(5):675-709.

25. Bruce V, Young AW. Understanding face recognition. Br J Psychol. 1986;77(3):305-27. 
26. Breen N, Caine D, Coltheart M. Models of Face Recognition and Delusional Misidentification: A Critical Review. Cogn Neuropsychol. 2000;17(1-3):55-71.

27. Breen N, Coltheart M, Caine D. A Two-Way Window on Face Recognition. Trends Cogn Sci. 2001;5(6):234-5.

28. Engell AD, Haxby JV, Todorov A. Implicit Trustworthiness Decisions: Automatic Coding of Face Properties in the Human Amygdala. J Cogn Neurosci. 20070 22;19(9):1508-19.

29. Todorov A, Gobbini MI, Evans KK, Haxby JV. Spontaneous retrieval of affective person knowledge in face perception. Neuropsychologia. 2007;45(1):163-73.

30. Todorov A, Pakrashi M, Oosterhof NN. Evaluating Faces on Trustworthiness After Minimal Time Exposure. Soc Cogn. 20090 1;27(6):813-33.

31. Willis J, Todorov A. First Impressions Making Up Your Mind After a 100-Ms Exposure to a Face. Psychol Sci. 2006 Jul 1;17(7):592-8.

32. Ellis HD, Lewis MB. Capgras delusion: a window on face recognition. Trends Cogn Sci. 2001 Apr 1;5(4):149-56.

33. Ellis HD, Young AW. Accounting for delusional misidentifications. Br J Psychiatry. 1990 Jan 8;157(2):239-48.

34. Edwards K, von Hippel W. Hearts and Minds: The Priority of Affective Versus Cognitive Factors in Person Perception. Pers Soc Psychol Bull. 1995 Oct 1;21(10):996-1011.

35. Pacilli MG, Mucchi-Faina A, Pagliaro S, Mirisola A, Alparone FR. When Affective (But Not Cognitive) Ambivalence Predicts Discrimination Toward a Minority Group. J Soc Psychol. 2013 Jan;153(1):10-24.

36. Bradley MM, Codispoti M, Cuthbert BN, Lang PJ. Emotion and motivation I: Defensive and appetitive reactions in picture processing. Emotion. 2001;1(3):276-98.

37. Lang PJ. The emotion probe: Studies of motivation and attention. Am Psychol. 1995;50(5):372-85.

38. Russell JA. A circumplex model of affect. J Pers Soc Psychol. 1980;39(6):1161-78.

39. Stangor C, Sullivan LA, Ford TE. Affective and Cognitive Determinants of Prejudice. Soc Cogn. 19910 1;9(4):359-80.

40. Abelson RP, Kinder DR, Peters MD, Fiske ST. Affective and semantic components in political person perception. J Pers Soc Psychol. 1982;42(4):619-30.

41. Başar E, Schmiedt-Fehr C, Öniz A, Başar-Eroğlu C. Brain oscillations evoked by the face of a loved person. Brain Res. 2008 Jun 12;1214:105-15. 
42. Denkova E, Botzung A, Scheiber C, Manning L. Implicit emotion during recollection of past events: A nonverbal fMRI study. Brain Res. 2006 Mar 17;1078(1):143-50.

43. Guerra P, Vico C, Campagnoli R, Sánchez A, Anllo-Vento L, Vila J. Affective processing of loved familiar faces: Integrating central and peripheral electrophysiological measures. Int J Psychophysiol. 2012 Jul 1;85(1):79-87.

44. Bate S, Cook SJ. Covert recognition relies on affective valence in developmental prosopagnosia: evidence from the skin conductance response. Neuropsychology. 2012 Sep;26(5):670-4.

45. Over $\mathrm{H}$, Cook R. Where do spontaneous first impressions of faces come from? Cognition. 20180 1;170:190-200.

46. R Core Team. R: A Language and environment for statistical computing. [Internet]. 2020. Available from: https://cran.r-project.org

47. The jamovi project. jamovi. 2021.

48. Koch A, Alves $\mathrm{H}$, Krüger $\mathrm{T}$, Unkelbach $\mathrm{C}$. A general valence asymmetry in similarity: Good is more alike than bad. J Exp Psychol Learn Mem Cogn. 2016;

49. R Core Team. R: A Language and environment for statistical computing. [Internet]. R Foundation for Statistical Computing, Vienna, Austria; 2021. Available from: https://www.R-project.org/

50. Young FW, Takane Y, Lewyckyj R. ALSCAL: A nonmetric multidimensional scaling program with several individual-differences options. Behav Res Methods Instrum. 1978 May 1;10(3):451-3.

51. Bates D, Maechler M. Matrix: Sparse and Dense Matrix Classes and Methods. R package version [Internet]. 2021. Available from: https://CRAN.R-project.org/package=Matrix

\section{Supplementary Files}

This is a list of supplementary files associated with this preprint. Click to download.

- appendix.docx 